\title{
Duskside F-region dynamo currents: its relationship with prereversal enhancement of vertical plasma drift
}

\author{
J. Park ${ }^{1,2}$, H. Lühr ${ }^{1}$, B. G. Fejer ${ }^{3}$, and K. W. Min ${ }^{2}$ \\ ${ }^{1}$ GFZ, German Research Center for Geosciences, Germany \\ ${ }^{2}$ Department of Physics, Korea Advanced Institute of Science and Technology, Korea \\ ${ }^{3}$ Center for Atmospheric and Space Science, Utah State University, USA
}

Received: 30 August 2010 - Accepted: 21 October 2010 - Published: 12 November 2010

\begin{abstract}
From magnetic field observations by CHAMP we estimate F-region dynamo current densities near the sunset terminator during solar maximum years from 2001 to 2002. The dynamo currents are compared with the pre-reversal enhancement (PRE) of vertical plasma drift as observed by ROCSAT-1. The seasonal-longitudinal variation of PRE can be largely related to the F-region dynamo current density, with the correlation coefficient reaching 0.74 . The correlation can be further improved if we consider a zonal gradient of the E-region Pedersen conductivity, which also depends on season and longitude. It is widely accepted that the Fregion dynamo drives PRE near sunset. For the first time, our observations provide confirmation for the close relationship between the F-region dynamo current density and PRE.
\end{abstract}

Keywords. Ionosphere (Electric fields and currents; Equatorial ionosphere; Ionosphere-atmosphere interactions)

\section{Introduction}

Thermospheric wind moving ionospheric plasma across the geomagnetic field generates an E-field by dynamo action, which is an important driver of the quiet-time equatorial ionosphere (e.g. Eccles, 1998a). After sunset the solar Extreme Ultra-Violet (EUV) flux, which is the main source of equatorial ionospheric plasma, becomes negligible. The ionospheric E-layer almost disappears due to rapid recombination while the F-region at higher altitudes persists. After sunset, consequently, the wind at F-region altitudes becomes an important driver for generating E-fields. The prereversal enhancement (PRE) of the upward plasma drift is generally attributed to this F-region dynamo acting on the

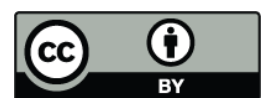

Correspondence to: J. Park

(park@gfz-potsdam.de) zonal conductivity gradient at the evening terminator (e.g. Heelis, 2004). However, the exact relationship between dynamo action and conductivity gradient is still an open issue (e.g. Eccles, 1998b). One of the reasons for little progress is the lack of observational studies on the dynamo current properties. Though PRE climatology has been extensively investigated for decades (e.g. Fejer et al., 1989, 2008; Scherliess and Fejer, 1999), few corresponding studies exist on the duskside F-region dynamo currents. Using geomagnetic field data of Magsat, Maeda et al. (1982) reported the existence of a hemispherically-symmetric meridional current system at dusk, which originates from currents induced by the equatorial F-region dynamo (Takeda and Maeda, 1983). From 5-year magnetic observations of the CHAMP satellite, Lühr and Maus (2006) showed that the F-region dynamo is strongest at noon and at dusk with opposite polarities, which was attributed to the change of zonal wind direction in the afternoon. Park et al. (2010) presented an extensive climatology of F-region dynamo currents both at noon and dusk. They focused on the noontime characteristics and detailed the wavenumber- 4 signatures. In this paper we will make use of CHAMP magnetic field data for outlining the general statistics of the duskside F-region dynamo current and its relation to the PRE.

\section{Observation}

The F-region dynamo driven by zonal neutral wind generates vertical currents at the equator, which are closed by symmetric meridional current systems in the two hemispheres (e.g. see Fig. 2 of Lühr and Maus, 2006). Hereafter, we will call the equatorial vertical current "F-region dynamo current." The current system generates zonal B-field deflections with opposite signs in the two hemispheres. In this paper we only consider this transverse magnetic component to investigate the F-region dynamo current. 

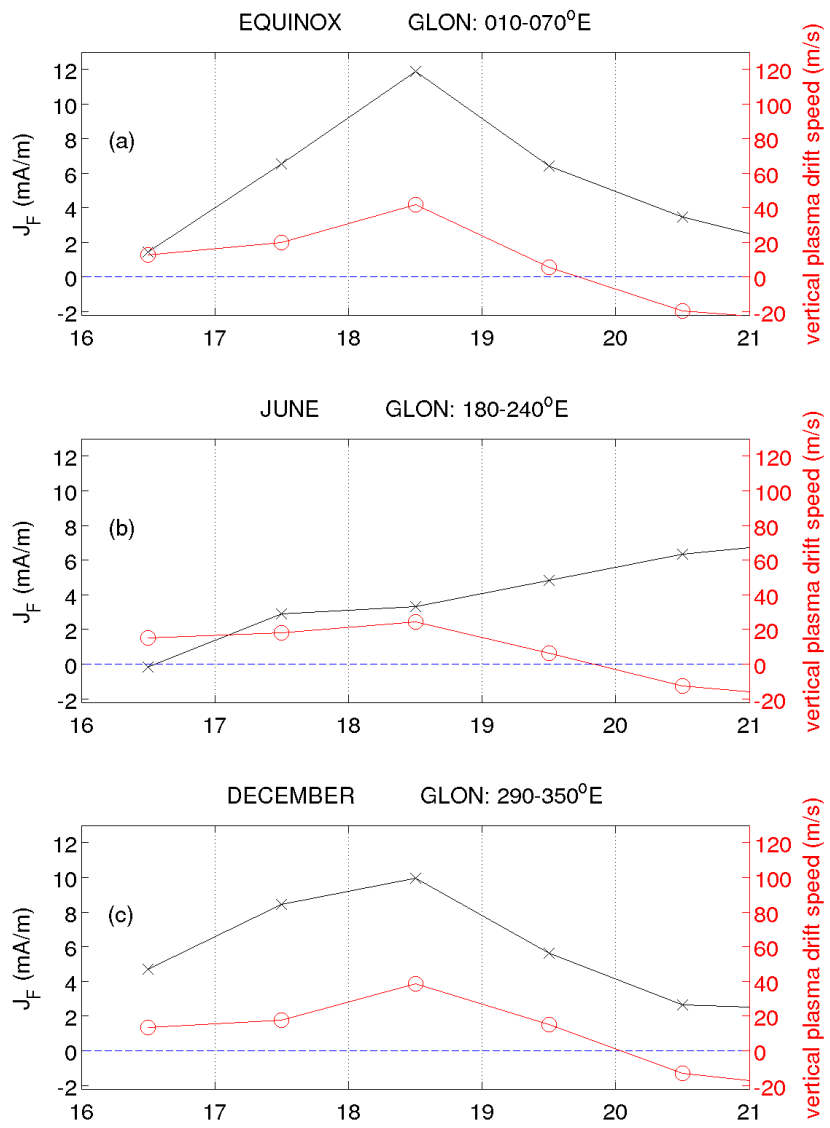

Fig. 1. Local time variation of the dynamo current density (black crosses) and vertical plasma drift (red circles) for the three seasons. For the equinox, June and December solstices only data in $10-70^{\circ}, 180-240^{\circ}$ and $290-350^{\circ}$ E GLON are considered, respectively, where the solar terminator is best aligned with the magnetic meridian and PRE amplitudes become large.

The Challenging Mini-Satellite Payload (CHAMP) has been observing geomagnetic field at altitudes of $300-450 \mathrm{~km}$ since 2000. Zonal deviation of the observed B-field from a reference model reflects meridional current systems; in this paper we use Pomme6 (http://www.geomag.us/models/ pomme6.html) as the reference model. We first decompose the latitudinal profile of zonal magnetic deflection by applying the Discrete Fourier Transform (DFT). If we add up latitudinally anti-symmetric terms, the field-line-integrated density of the F-region dynamo current, $J_{\mathrm{F}}$, can be computed from the peak-to-peak amplitude of the anti-symmetric variation, $\Delta B_{\mathrm{y}}$ (Eq. 2 of Lühr and Maus, 2006):

$J_{\mathrm{F}}=\frac{1}{\mu_{0}} \Delta B_{\mathrm{y}}$,

where $\mu_{0}$ is the permeability of free space. For more details on the retrieval of the F-region dynamo currents from magnetic field observation, readers are referred to Park et al. (2010). But, there are four notable differences with re- spect to this work. First, Park et al. (2010) used zonal magnetic deflection within $\pm 40^{\circ}$ magnetic latitude (MLAT), and the bipolar peaks sometimes appeared beyond $\pm 15^{\circ}$ MLAT. In such cases the magnetic signature was attributed to other sources than the equatorial F-region dynamo currents, whose density could not be deduced. In this work we narrowed the latitude range to $\pm 20^{\circ}$ MLAT to avoid this problem. Second, Park et al. (2010) averaged zonal magnetic deflections within $24^{\circ}$-wide geographic longitude (GLON) cells. In this study we use overlapping $30^{\circ}$-wide GLON cells at $15^{\circ}$ separation, as Fejer et al. (2008) did for interpreting the vertical drift data of ROCSAT-1. Finer longitude cells might be useful to investigate smaller-scale variations, but concomitant poor statistics in each cell can be contaminated by artifacts. Third, while Park et al. (2010) used data from 2001 to 2004, here we concentrate on years of high solar activity (i.e. active PRE) from 2001 to 2002. Fourth, residual field whose magnitude is larger than $30 \mathrm{nT}$ is neglected while the threshold was $50 \mathrm{nT}$ in Park et al. (2010). For local time (LT) distribution we use 1-h bins to average the data.

Figure 1 depicts the LT variation of the F-region dynamo current density (black) during three seasons (equinox: March-April and September-October, June solstice: MayAugust, and December solstice: November-February). Positive (negative) currents flow upward (downward) at the dip equator. Vertical plasma drift velocity (red) derived from ROCSAT- 1 measurements at $600 \mathrm{~km}$ altitude for $F_{10.7}=170$ (Fejer et al., 2008) are overlaid. Positive (negative) velocity corresponds to the upward (downward) plasma drift at the dip equator. For the equinox, June and December solstices only data in $10-70^{\circ}$ (negligible magnetic declination), 180$240^{\circ}$ (positive magnetic declination) and $290-350^{\circ} \mathrm{E}$ (negative magnetic declination) GLON are considered, respectively, where the best alignment of the solar terminator with the magnetic meridian is accomplished and PRE amplitudes become large. The F-region dynamo current (black) reaches a large value around sunset (between 17:00 and 19:00 LT) when the vertical drift (red) maximizes. It is interesting to note that the F-region dynamo current density remains at sizable positive values beyond the time when the vertical drift turns negative. Especially, the magnetic signatures during June solstice even exhibit a slight increase after 19:00 LT. Near June solstice, both the F-region dynamo currents around sunset and PRE maximum are smaller than during the other seasons.

Figure 2 shows the longitudinal variations of the F-region dynamo current density (black crosses: estimated from CHAMP observations) for each season. PRE maximum velocity derived from ROCSAT-1 measurements for $F_{10.7}=$ 170 (Fejer et al., 2008) are given as red circles. CHAMP observations between 17:00 and 19:00 LT are used here. The average index of the solar radio flux $\left(F_{10.7}\right)$ is given in the heading for each season. First, the average levels of F-region dynamo current and PRE are largest during equinoxes and smallest during June solstice. However, the longitudinal 

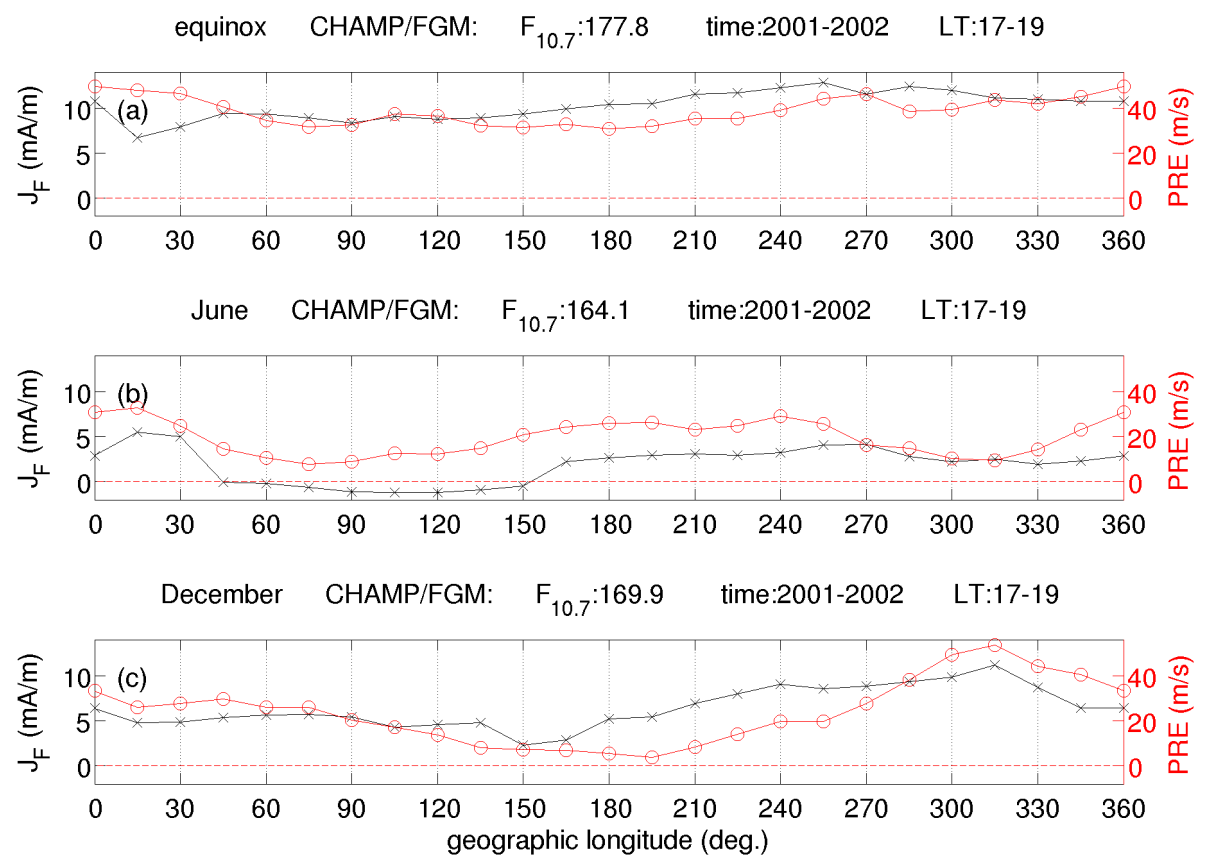

Fig. 2. Seasonal-longitudinal distribution of the F-region dynamo current density (black crosses) and PRE peak velocity (red circles). Each panel corresponds to a different season. Positive (negative) current density denotes upward (downward) current at the dip equator.

variations are quite different from season to season. For example, during equinoxes both the PRE and the dynamo current density exhibit small longitudinal variations. During June solstice the general trends of PRE and dynamo current follow each other, but the peak longitudes of PRE are slightly displaced from those of the dynamo current. Around December solstice both PRE and dynamo current attain their maximum in the western longitude regions $\left(210-360^{\circ} \mathrm{E}\right)$ and the minimum around $180^{\circ} \mathrm{E}$.

\section{Discussion}

First of all, we want to discuss the LT dependence of the Fregion dynamo current shown in Fig. 1. Zonal neutral wind in the ionospheric F-region, as described above, drives vertical current in the equatorial ionosphere (e.g. see Fig. 10 of Heelis, 2004). Let us assume a simple model where zonal current divergence is negligible. Such a current model, though simple, is widely used (Heelis, 2004; Kelley, 2009) and apt to give physical intuition on the F-region dynamo. In the model meridional divergence of the F-region dynamo current generates field-aligned currents (FACs) which are closed by meridional currents at conjugate E-regions. The conductivity of the E-region controls polarization E-fields in the meridional (outward/inward) direction. When the Eregion conductivity is much higher (lower) than the F-region conductivity, polarization E-field becomes low (high) just like in a shorted (an open) circuit. Combining Eqs. (10-11) of Heelis (2004) or Eqs. (3.10-11) of Kelley (2009), we get equations for the polarization E-field and the F-region dynamo currents:

$$
\begin{aligned}
& \boldsymbol{E}=\frac{\Sigma_{\mathrm{P}}^{\mathrm{F}}}{\Sigma_{\mathrm{P}}^{\mathrm{E}}+\Sigma_{\mathrm{P}}^{\mathrm{F}}} \boldsymbol{u} \times \boldsymbol{B}, \\
& J_{\mathrm{F}}=\Sigma_{\mathrm{P}}^{\mathrm{E}} E=\frac{\Sigma_{\mathrm{P}}^{\mathrm{E}} \Sigma_{\mathrm{P}}^{\mathrm{F}}}{\Sigma_{\mathrm{P}}^{\mathrm{E}}+\Sigma_{\mathrm{P}}^{\mathrm{F}}} u B,
\end{aligned}
$$

where $\boldsymbol{u}$ is neutral wind velocity, $\boldsymbol{B}$ the ambient magnetic field, $\Sigma_{\mathrm{P}}^{\mathrm{E}}\left(\Sigma_{\mathrm{P}}^{\mathrm{F}}\right)$ field-line-integrated E-(F-)region Pedersen conductivity, $u$ the zonal wind speed, and $B$ the horizontal strength of the ambient magnetic field. According to Eq. (2), the meridional polarization E-field is enhanced after sunset. The zonal gradient of the meridional E-field, which in turn affects the zonal E-field through its irrotational nature, is believed to be the main driver of PRE (Murphy and Heelis, 1986; Eccles, 1998a). From Eqs. (12) and (20) of Eccles (1998a), we can get the following equation near the F-peak altitude ( $\sim 400 \mathrm{~km}$ altitude) around sunset:

$v_{L} \propto \frac{\partial v_{\phi}}{\partial \phi}$,

where $v_{L}$ is the meridional (outward/inward) plasma drift speed (due to the zonal E-field causing PRE), $v_{\phi}$ is the zonal plasma drift speed (due to the meridional E-field given by Eq. 2), and $\phi$ the azimuth angle along the zonal direction (positive eastward). Combining Eqs. (2) and (4) yields:

$$
v_{L}=A \frac{\partial v_{\phi}}{\partial \phi}=A \frac{\partial}{\partial \phi}\left(\frac{\Sigma_{\mathrm{P}}^{\mathrm{F}}}{\Sigma_{\mathrm{P}}^{\mathrm{E}}+\Sigma_{\mathrm{P}}^{\mathrm{F}}} u\right),
$$



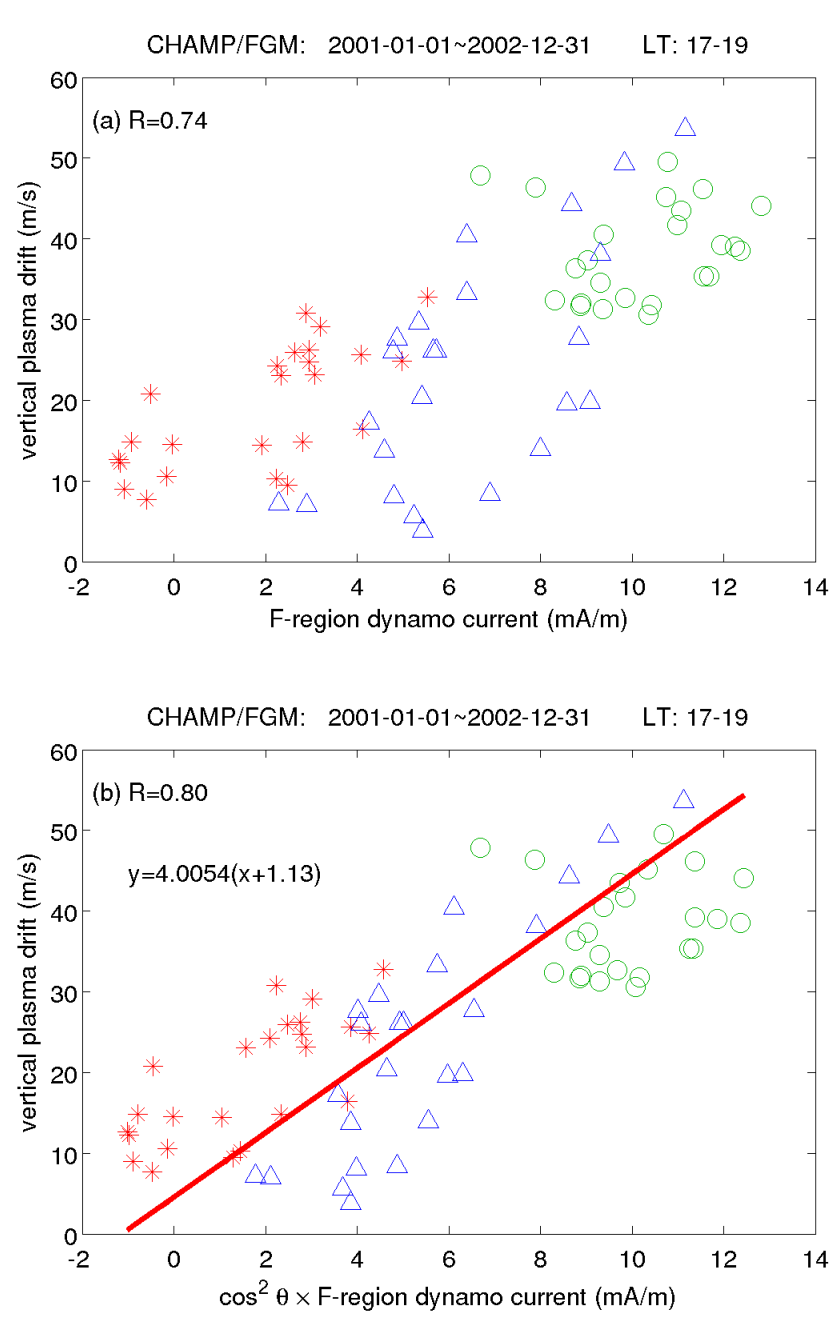

Fig. 3. Relation between PRE and (a) F-region dynamo current, and (b) F-region dynamo current multiplied by assumed conductivity gradients. Data points are the same as in Fig. 2. Circles, asterisks, and triangles represent equinox, June solstice, and December solstice readings, respectively.

where $A$ is a proportionality constant. The zonal gradient of $\Sigma_{\mathrm{P}}^{\mathrm{F}}$ is smaller than that of $\Sigma_{\mathrm{P}}^{\mathrm{E}}$ around sunset (Farley et al., 1986). Also, $u$ depends only weakly on $\phi$ (e.g. see Fig. 3 of Liu et al., 2006, and Fig. 2 of Liu et al., 2009). Under these conditions Eq. (5) can be manipulated as:

$$
\begin{aligned}
v_{L} & \approx A \Sigma_{\mathrm{P}}^{\mathrm{F}} u \frac{\partial}{\partial \phi}\left(\frac{1}{\Sigma_{\mathrm{P}}^{\mathrm{E}}+\Sigma_{\mathrm{P}}^{\mathrm{F}}}\right)=-\frac{A \Sigma_{\mathrm{P}}^{\mathrm{F}} u}{\left(\Sigma_{\mathrm{P}}^{\mathrm{E}}+\Sigma_{\mathrm{P}}^{\mathrm{F}}\right)^{2}} \frac{\partial}{\partial \phi}\left(\Sigma_{\mathrm{P}}^{\mathrm{E}}+\Sigma_{\mathrm{P}}^{\mathrm{F}}\right) \\
& \approx-\frac{A \Sigma_{\mathrm{P}}^{\mathrm{F}} u}{\left(\Sigma_{\mathrm{P}}^{\mathrm{E}}+\Sigma_{\mathrm{P}}^{\mathrm{F}}\right)^{2}} \frac{\partial \Sigma_{\mathrm{P}}^{\mathrm{E}}}{\partial \phi}
\end{aligned}
$$

According to Eq. (6) of Lühr and Maus (2006) $\Sigma_{\mathrm{P}}^{\mathrm{F}} u$ can be estimated as $J_{\mathrm{F}}^{\text {day }} / B$, where $J_{\mathrm{F}}^{\text {day }}$ signifies $J_{\mathrm{F}}$ on the dayside (before sunset). As $\Sigma_{\mathrm{P}}^{\mathrm{F}} u$ depends weakly on LT, this estimation can be directly applied to Eq. (6):
$v_{L} \approx-\frac{A J_{\mathrm{F}}^{\text {day }}}{B\left(\Sigma_{\mathrm{P}}^{\mathrm{E}}+\Sigma_{\mathrm{P}}^{\mathrm{F}}\right)^{2}} \frac{\partial \Sigma_{\mathrm{P}}^{\mathrm{E}}}{\partial \phi}$,

This equation shows that the plasma drift component, $v_{L}$, corresponding to PRE is determined by (a) the pre-sunset dynamo current strength and (b) local-time gradient of the Eregion conductivity. Note again that $v_{L}$ in Eq. (7) is vertical plasma drift speed near $400 \mathrm{~km}$ altitude. Pingree and Fejer (1987) showed that vertical gradient of vertical plasma drift speed is $\sim 0.01 \mathrm{~m} / \mathrm{s} / \mathrm{km}$ on average. The difference of vertical drift speed between $400 \mathrm{~km}$ and $600 \mathrm{~km}$ altitudes is $\sim 2 \mathrm{~m} / \mathrm{s}$, which is smaller than seasonal/longitudinal variation of Fejer et al. (2008) model. Therefore, $v_{L}$ in Eq. (7) can be reasonably approximated by ROCSAT- 1 observations at $600 \mathrm{~km}$.

In Fig. 3a the PRE peak velocities are plotted versus the F-region dynamo current density during 17:00-19:00 LT, which we consider as representing the pre-sunset F-region dynamo current. Note that the current densities given in Fig. 1 do not vary drastically during 17:00-19:00 LT. Each data point in Fig. 3a corresponds to a longitude-bin average from Fig. 2. Circles, asterisks, and triangles represent equinox, June solstice, and December solstice readings, respectively. The correlation coefficient between the two parameters is reasonably high (0.74).

Next, let us consider the zonal gradient of the E-region conductivity. The maximum zonal gradient is attained in case of a perfect alignment of magnetic meridian and sunset terminator (Tsunoda, 1985). The zonal gradient decreases with an increase of this angle, due to the high plasma mobility along the B-field lines. As an initial guess, let us choose a cosine function for describing the zonal conductivity gradient:

$-\frac{\partial \Sigma_{\mathrm{P}}^{\mathrm{E}}}{\partial \phi} \sim \cos ^{2} \theta$,

where $\theta$ is the angle between the solar terminator and geomagnetic meridian. Note that $\theta$ depends on season (solar terminator angle) and longitude (geomagnetic declination). In Fig. 3b F-region dynamo currents multiplied by the assumed conductivity gradient (Eq. 8) are considered. The red line is the result of linear regression. The correlation between the two quantities is higher in panel (b) than in panel (a) though the improvement is not large. We get compatible correlation results if we use other power values (1 or 3) for $\cos \theta$. They change the steepness of the cosine curves. Figure 3 implies that PRE amplitude is largely determined by the presunset F-region dynamo current density, and that E-region conductivity gradient at the dusk terminator can further enhance PRE. Note, however, that Eq. (7) has been derived by a number of assumptions. More thorough theoretical work with realistic inputs is warranted to elucidate the exact relationship between PRE and the F-region dynamo. 


\section{Summary}

From magnetic field observations by CHAMP we estimated the pre-sunset F-region dynamo current densities and compared them with PRE velocities given in Fejer et al. (2008). Main results can be summarized as follows:

1. The seasonal variation of PRE is consistent with that of the pre-sunset F-region dynamo current strength. Both are largest during equinoxes and smallest during June solstice.

2. The seasonal/longitudinal variations of PRE can also be largely explained by the pre-sunset F-region dynamo currents $(R=0.74)$.

3. The correlation can be further improved if we consider a zonal gradient of the E-region Pedersen conductivity, as resulting from the irrotational nature of the E-field near the dusk terminator (Eccles, 1998a).

It is widely accepted that the F-region dynamo drives the pre-reversal enhancement of vertical plasma drift (PRE) near sunset. For the first time, our observations provide confirmation for the close relationship between the F-region dynamo current density and PRE.

Acknowledgements. The CHAMP mission is sponsored by the Space Agency of the German Aerospace Center (DLR) through funds of the Federal Ministry of Economics and Technology, following a decision of the German Federal Parliament (grant code 50EE0944). The data retrieval and operation of the CHAMP satellite by the German Space Operations Center (GSOC) is acknowledged.

Topical Editor K. Kauristie thanks one anonymous referee for her/his help in evaluating this paper.

\section{References}

Eccles, J. V.: A simple model of low-latitude electric fields, J. Geophys. Res., 103(A11), 26699-26708, 1998a.

Eccles, J.: Modeling investigation of the evening prereversal enhancement of the zonal electric field in the equatorial ionosphere, J. Geophys. Res., 103(A11), 26709-26719, 1998b.

Farley, D. T., Bonelli, E., Fejer, B. G., and Larsen, M. F.: The Prereversal Enhancement of the Zonal Electric Field in the Equatorial Ionosphere, J. Geophys. Res., 91(A12), 13723-13728, 1986.
Fejer, B. G., de Paula, E. R., Batista, I. S., Bonelli, E., and Woodman, R. F.: Equatorial F Region Vertical Plasma Drifts During Solar Maxima, J. Geophys. Res., 94(A9), 12049-12054, 1989.

Fejer, B. G., Jensen, J. W., and Su, S.-Y.: Quiet time equatorial $\mathrm{F}$ region vertical plasma drift model derived from ROCSAT-1 observations, J. Geophys. Res., 113, A05304, doi:10.1029/2007JA012801, 2008.

Heelis, R. A.: Electrodynamics in the low and middle latitude ionosphere: a tutorial, J. Atmos. Solar Terr. Phys., 66, 825-838, 2004.

Kelley, M. C.: The Earth's Ionosphere: Plasma Physics and Electrodynamics, 2nd edition, Elsevier, London, 2009.

Liu, H., Lühr, H., Watanabe, S., Köhler, W., Henize, V., and Visser, P.: Zonal winds in the equatorial upper thermosphere: Decomposing the solar flux, geomagnetic activity, and seasonal dependencies, J. Geophys. Res., 111, A07307, doi:10.1029/2005JA011415, 2006.

Liu, H., Watanabe, S., and Kondo, T.: Fast thermospheric wind jet at the Earth's dip equator, Geophys. Res. Lett., 36, L08103, doi:10.1029/2009GL037377, 2009.

Lühr, H. and Maus, S.: Direct observation of the F region dynamo currents and the spatial structure of the EEJ by CHAMP, Geophys. Res. Lett., 33, L24102, doi:10.1029/2006GL028374, 2006.

Maeda, H., Iyemori, T., Araki, T., and Kamei, T.: New evidence of a meridional current system in the equatorial ionosphere, Geophys. Res. Lett., 9(4), 337-340, 1982.

Murphy, J. A. and Heelis, R. A.: Implications of the Relationship Between Electromagnetic Drift Components at Mid and Low Latitudes, Planet. Space Sci., 34, 645-652, 1986.

Park, J., Lühr, H., and Min, K. W.: Characteristics of F-region dynamo currents deduced from CHAMP magnetic field measurements, J. Geophys. Res., 115, A10302, doi:10.1029/2010JA015604, 2010.

Pingree, J. E. and Fejer, B. G.: On the Height Variation of the Equatorial F Region Vertical Plasma Drifts, J. Geophys. Res., 92(A5), 4763-4766, 1987.

Scherliess, L. and Fejer, B.: Radar and satellite global equatorial F region vertical drift model, J. Geophys. Res., 104(A4), 6829$6842,1999$.

Takeda, M. and Maeda, H.: F-region dynamo in the evening. interpretation of equatorial D anomaly found by Magsat, J. Atmos. Terr. Phys., 45, 401-408, 1983.

Tsunoda, R.: Control of the Seasonal and Longitudinal Occurrence of Equatorial Scintillations by the Longitudinal Gradient in Integrated E Region Pedersen Conductivity, J. Geophys. Res., 90(A1), 447-456, 1985. 